\title{
Submucosal Injection Solutions for ESD: Separating the Winners from the Losers
}

\author{
Toshio Kuwai ${ }^{1} \cdot$ Yuzuru Tamaru $^{1} \cdot$ Ryusaku Kusunoki ${ }^{1} \cdot$ Sauid Ishaq ${ }^{2,3}$
}

Published online: 30 July 2019

(c) Springer Science+Business Media, LLC, part of Springer Nature 2019

\begin{abstract}
Although endoscopic submucosal dissection (ESD), developed in Japan in late 1990s, is now increasingly adopted worldwide for en bloc resection of superficial tumors, including early colorectal neoplasms in the upper and lower gastrointestinal tracts [1,2], adoption of colorectal ESD has not been widespread due to technical challenges and limitations imposed by the complex anatomic features of the large intestine such as acute angulation, a relatively thinner wall, and a narrow lumen/operating space [3]. Submucosal injection of a solution, by separating the mucosal and muscular layers, is the most important step of ESD, enabling safe circumferential mucosal incision and submucosal dissection. A well-formed submucosal fluid cushion is an essential requirement in order to maintain sufficient mucosal elevation to lift lesions during ESD, considered one of the most important factors used to improve safety and outcomes [4, 5]. Lengthier ESD requires a durable submucosal cushion for lasting elevation in order to achieve safe dissection. At this point, we would like to discuss an important controversy addressing the choice of ideal solution, defined as one that provides a sufficiently wide and durable submucosal cushion, is easy to inject, is widely available, and is harmless to tissue and the host. Since there is no perfect solution, several kinds of submucosal injection solutions have been developed globally [4-8].
\end{abstract}

The most important requisites of an optimal injection solution are provision of a thick and lasting submucosal cushion with durable lesion elevation and good preservation of the tissue specimen facilitating accurate histopathologic

Toshio Kuwai

kuwai.toshio.ur@mail.hosp.go.jp

1 Department of Gastroenterology, National Hospital Organization, Kure Medical Center and Chugoku Cancer Center, 3-1 Aoyama-cho, Kure 737-0023, Japan

2 Gastroenterology Department, Dudley Group Hospitals, Birmingham City University, Birmingham, UK

3 St. George's University, St. George's, Grenada staging. Although normal saline (NS) is the most widely used solution as it is simple to use and cheap, the cushion created with NS is sustained for a very short period due to rapid resorption of fluid and sodium requiring repeated injections which may be problematic for effective ESD. To overcome these limitations, several alternative submucosal injection solutions have been developed and studied consisting of polymers, emulsions, and charged molecules that are more viscous and less easily resorbed than saline. Out of all available submucosal injection solutions such as physiological saline, hypertonic saline, and glucose, glycerin/fructose $/ 0.4 \%$ sodium hyaluronate $(\mathrm{SH})$ was the only solution for submucosal injection approved in Japan to date. Therefore, though it is the most commonly used submucosal injection solution for ESD in Japan [4, 5], there are recent concerns that $\mathrm{SH}$ might promote cancer cell proliferation [10]. To mitigate this serious concern, a new submucosal injection solution consisting of $0.6 \%$ sodium alginate (SA) was made available in Japan in June 2019. SA is used in clinical practice to treat peptic ulcer or used as hemostatic agent. SA has superior mucosal elevation than $0.4 \% \mathrm{SH}$. In another study, its clinical efficacy was reported as noninferior to $0.6 \%$ SA [6]. Therefore, the use of SA as a submucosal injection should increase in clinical practice. In this study, EndoClot ${ }^{\circledR}$ SIS polysaccharide (principal ingredient sodium carboxymethyl starch) provided longer-lasting elevation than SA. One of the strengths of this study was that both in vivo and in vitro experiments were conducted to verify safety and effectiveness.

In this issue of Digestive Diseases and Sciences, Dai et al. [9] evaluated the feasibility and safety of injected EndoClot ${ }^{\circledR}$ SIS polysaccharide as a submucosal injection solution for ESD of lesions in the porcine stomach compared with sigMAVisc $^{\mathrm{TM}}$ (sodium hyaluronate) or Eleview ${ }^{\mathrm{TM}}$ (medium chain triglycerides/poloxamer 188/polyoxyl-15-hydroxystearate). The authors reported increased effective separation of the mucosa and muscular layers and reduced adverse events, in particular bleeding, in comparison with sigMAVisc ${ }^{\mathrm{TM}}$ 
or Eleview ${ }^{\mathrm{TM}}$ in ex vivo and in vivo ESD studies, with the authors concluding that EndoClot ${ }^{\circledR}$ SIS polysaccharide is an effective submucosal injection solution.

Despite the encouraging result that EndoClot ${ }^{\circledR}$ SIS polysaccharide provided a longer-lasting result with sufficient elevation, there are unresolved issues that need addressing before the clinical usefulness of EndoClot ${ }^{\circledR}$ SIS polysaccharide is established. First, endoscopic ultrasonography performed in this study showed that EndClot ${ }^{\circledR}$ SIS polysaccharide forms a viscous gel after submucosal injection. In my experience, there is difficulty with cutting and dissecting the gel form of EndoGel (i.e., ESD training model) [11]. We are also aware that the gel interferes with the procedure to a great extent. Such interference in ESD with the presence of gel in the submucosal layer may pose difficulty to identifying the appropriate level of the submucosa (just above the muscular layer for gastric lesions) that potentially could jeopardize accurate and safe dissection. To resolve this issue, histopathologic examination of ESD specimens should be performed to confirm that specimens were dissected at an appropriate level (layer). Second, regarding ESD outcomes, a significant advantage of EndoClot ${ }^{\circledR}$ SIS polysaccharide versus the other solutions could not be shown in the in vivo study. Since this outcome may have been affected by the small sample size as described by the authors in their discussion of limitations, further verification with a larger sample size is necessary. Furthermore, ESD outcomes with EndoClot ${ }^{\circledR}$ SIS polysaccharide under difficult situations (such as in difficult locations or in the presence of fibrosis) would be useful to ascertain its usefulness in challenging situations. Third, since only the results of experimental ex vivo and in vivo experiments were shown, clinical trials are required prior to approval for use in clinical practice.

Despite these caveats, EndoClot ${ }^{\circledR}$ SIS polysaccharide has the potential to be a contender to be included in as a useful submucosal injection solution. But to become the preferred solution, larger head-to-head studies in humans are needed to prove its superiority over widely used solutions such as with SH and SA.

Acknowledgments The authors thank Naoko Matsumoto for assistance in administrative support.

\section{Compliance with Ethical Standards}

Conflict of interest The authors declare that they have no conflict of interest.

\section{References}

1. Kuwai T, Yamaguchi T, Imagawa H, et al. Endoscopic submucosal dissection of early colorectal neoplasms with a monopolar scissor-type knife: short- to long-term outcomes. Endoscopy. 2017;49:913-918.

2. Kuwai T, Yamaguchi T, Imagawa $\mathrm{H}$, et al. Endoscopic submucosal dissection for early esophageal neoplasms using the stag beetle knife. World J Gastroenterol. 2018;24:1632-1640.

3. Ferlitsch M, Moss A, Hassan C, et al. Colorectal polypectomy and endoscopic mucosal resection (EMR): European Society of Gastrointestinal Endoscopy (ESGE) clinical guideline. Endoscopy. 2017;49:270-297.

4. Yamamoto H, Yahagi N, Oyama T, et al. Usefulness and safety of $0.4 \%$ sodium hyaluronate solution as a submucosal fluid "cushion" in endoscopic resection for gastric neoplasms: a prospective multicenter trial. Gastrointest Endosc. 2008;67:830-839.

5. Hirasaki S, Kozu T, Yamamoto H, et al. Usefulness and safety of $0.4 \%$ sodium hyaluronate solution as a submucosal fluid "cushion" for endoscopic resection of colorectal mucosal neoplasms: a prospective multi-center open-label trial. BMC Gastroenterol. 2009;9:1.

6. Uemura N, Oda I, Saito Y, et al. Efficacy and safety of $0.6 \%$ sodium alginate solution in endoscopic submucosal dissection for esophageal and gastric neoplastic lesion: a randomized controlled study. Dig Endosc. 2019;31(4):396-404. https://doi.org/10.1111/ den. 13352.

7. Spadaccini M, Hassan C, Maselli R, et al. Efficacy and safety of SIC-8000 (Eleview(R)) for submucosal injection for endoscopic mucosal resection and endoscopic submucosal dissection in an in vivo porcine model. Dig Liver Dis. 2018;50:260-266.

8. Wedi E, Koehler P, Hochberger J, et al. Endoscopic submucosal dissection with a novel high viscosity injection solution (LiftUp) in an ex vivo model: a prospective randomized study. Endosc Int Open. 2019;7:E641-E646.

9. Dai M-S, Hu K-W, Wu W, Yin G-J, Hu D-M. EndoClot ${ }^{\circledR}$ SIS polysaccharide injection as a submucosal fluid cushion for endoscopic mucosal therapies: results of ex vivo and in vivo studies. Dig Dis Sci. (Epub ahead of print). https://doi.org/10.1007/s10620-01905686-4.

10. Matsui Y, Inomata M, Izumi K, Sonoda K, Shiraishi N, Kitano $S$. Hyaluronic acid stimulates tumor-cell proliferation at wound sites. Gastrointest Endosc. 2004;60:539-543.

11. Mizuno K, Sato H, Hashimoto S, Sato Y, Terai S. A novel training model composed of nonbiological materials for endoscopic submucosal dissection. Gastrointest Endosc. 2016;84:373-374.

Publisher's Note Springer Nature remains neutral with regard to jurisdictional claims in published maps and institutional affiliations. 